\title{
The Anisotropy in the Distribution of the Major Axes of Elongated Shakhbazian CGCG
}

\author{
R.A. Vardanian \\ Byurakan Astrophysical Observatory, Byurakan, 378433, Armenia
}

In 1978 we have shown that Shakhbazian CGCG have an elongated form (Vardanian \& Melik-Alaverdian 1978). Morever, they turned out to be of comet forms (1996), at the head of which basically are situated their bright members. This enables determination with high accuracy of the position angles $\theta$ of major axes of the elongated Shakhbazian CGCG. A closer examination of these angles shows that distribution of orientations of the major axes of groups is anisotropic and has two maximums (Vardanian 1996). (See Table, where $\theta$ is position angle, $\mathrm{N}$ is number of CGCG). These maximums are oriented in mutually perpendicular directions $\left(\theta=45^{\circ}\right.$ and $\left.\theta=135^{\circ}\right)$.

\begin{tabular}{ccccc} 
No & $\theta$ & $N$ & $\theta$ & $N$ \\
\hline 1 & $0^{\circ}-10^{\circ}$ & 3 & $90^{\circ}-100^{\circ}$ & 6 \\
2 & $10-20$ & 6 & $100-110$ & 11 \\
3 & $20-30$ & 11 & $110-120$ & 15 \\
4 & $30-40$ & 14 & $120-130$ & 13 \\
5 & $40-50$ & 27 & $130-140$ & 25 \\
6 & $50-60$ & 16 & $140-150$ & 14 \\
7 & $60-70$ & 15 & $150-160$ & 10 \\
8 & $70-80$ & 8 & $160-170$ & 9 \\
9 & $80-90$ & 3 & $170-180$ & 4
\end{tabular}

\section{References}

Vardanian, R.A., \& Melik-Alaverdian, Yu. K. 1978, Astrofizika, 14, 195

Vardanian, R. A. 1996, Astrofizika, 39, 585 